\title{
Impact of Vibration Models on Heat Capacities of HF Molecular System
}

\author{
Guo Yue Liu, Ying Wu, Bi Tao Liao \\ Research Center of Computational Physics, Mianyang Normal University, Mianyang, China \\ Email: guoyueliu@sohu.com
}

Received May 2014

\begin{abstract}
Based on the analysis of completeness and finiteness of HF molecular vibrational levels, HF systemic vibrational heat capacity is studied with quantum statistical and full set of vibrational energy level determined AM (algebraic method). The results show that correct vibrational description and vibrational energy level set of HF system are key factors in calculating heat capacity, HF heat capacity data determined by AM energy spectra $\left\{E_{v}\right\}$ are much superior to the ones of harmonic oscillator model, $\mathrm{AM}$ results are agreement with experiment data.
\end{abstract}

\section{Keywords}

\section{HF Molecule, Vibrational Energy Level, Quantum Ensemble, Molar Heat Capacity}

\section{Introduction}

It is well known that macroscopic thermodynamic system is consists of many molecules, vibration is molecular intrinsic movement. Molecular vibration directly effects for systematic macroscopic thermodynamic properties (TDP), for example, affect the heat capacity of actual gas. Diatomic molecular vibration makes contributions for thermodynamic properties of macroscopic systems, the problems have been the important topics worth studying, there are some meaningful results [1] [2].

How to deal with diatomic molecular vibrational energy is important and difficult to solve the thermodynamic properties (TDP) using statistical methods. For example, diatomic molecular vibration was seen as a simple harmonic vibration, quantum summation was usual over $0 \rightarrow \infty$ in computing vibrational partition function (VPF) [3]. But diatomic molecular is not simple harmonic oscillator (SHO), there is actually not infinite vibrational levels, too. Important SHO heat capacity cannot show reasonability in wider temperature range. The potential energy function (PEF) with good physical behavior should be necessary. Morse function [4], MS potential (Murrell-Sorbie) [5], ECM potential (energy-and consistent-method) [6] [7], and so on, they are all good analytical potential energy functions. In 1932, Dunham proposed an analytical diatomic molecular vibration-rotation level formula [8]. Since high order vibration coefficients and the number of vibrational levels are difficult to be determined, so far the thermodynamic work report has not been seen. In 1953, Herzberg proposed an important vibration level formula, coefficients or levels can be directly determined by related vibration spectrum data [9]. Since the selected number of expansion item is uncertainty and the analytical expression is more 
complexity, the work used Herzberg's vibrational levels has not been seen, too.

Once the number of vibrational levels is unlimited, the paradox will be inevitable that the vibration energy level can be greater than the diatomic molecular dissociation energy. It is obvious that accuracy and finiteness of vibrational energy spectrum (a full set of vibrational levels) are essential to study on macroscopic thermodynamic properties (TDP). Ab initio should be a meaningful way to directly obtain numerical vibration levels. But there are differences between $a b$ initio levels and experiment data, the differences will rise as vibrational quantum number increases [10]. There are some experiment data like RKR data [11]-[13], too. But such data are often difficult to give high vibrational levels and the highest vibration quantum number.

In this work, the algebraic method (AM) proposed by Sun Weiguo [14] is adopted to obtain a full set of vibration energy level and maximum quantum number of HF molecular in ground state. Using quantum statistical ensemble theory, the statistical contributions for vibration heat capacity of HF gas are discussed.

\section{The Full Set for Vibrational Energy Level of HF in Ground State $\mathbf{X}^{1} \Sigma^{+}$}

The ground state $\mathrm{X}^{1} \Sigma^{+}$of hydrogen fluoride is particularly well characterized by spectroscopic methods and very precise molecular constants for the lower vibrtional levels are available. HF is important for high - efficiency chemical lasers in the infrared (IR) and far infrared (FIR), and the ground state pure rotational transitions of HF serve as accurate wavelength standards for IR and FIR spectroscopy [15]. In 1975, W. Meyer and P. Rosmus used coupled electronic pair approach (CEPA) method to study the spectra data and the potential energy behavior of HF molecule in ground state and given dissociation energy $D e=5.83 \mathrm{eV}$ [16]. In 1990, J. A. Coxon et al. studied the vibration energy levels of HF in ground state, 20 vibrational levels were given, and at the vibrational quantum number $v=19$, relative vibrational level is $49026.508 \mathrm{~cm}^{-1}$ [15], but the number of vibrational energy levels in the ground state of HF did not give.

The high order expansion of diatomic molecular vibration level was derived by Sun Weiguo and his collaborators using second order perturbation theory [14].

$$
\begin{aligned}
& E_{v}=\omega_{0}+\left(\omega_{e}+\omega_{e 0}\right)\left(v+\frac{1}{2}\right)-\omega_{e} x_{e}\left(v+\frac{1}{2}\right)^{2} \\
& +\omega_{e} y_{e}\left(v+\frac{1}{2}\right)^{3}+\omega_{e} z_{e}\left(v+\frac{1}{2}\right)^{4}+\omega_{e} t_{e}\left(v+\frac{1}{2}\right)^{5} \\
& +\omega_{e} s_{e}\left(v+\frac{1}{2}\right)^{6}+\omega_{e} r_{e}\left(v+\frac{1}{2}\right)^{7}+\cdots
\end{aligned}
$$

where $v$ is vibrational quantum number, $\omega_{0}, \omega_{0 e}, \omega_{e}, \omega_{e} x_{e}, \omega_{e} y_{e}, \omega_{e} x_{e}, \omega_{e} x_{e}, \omega_{e} x_{e}$ and $\omega_{e} x_{e}$ are molecular vibrational spectroscopy data, $\omega_{0}$ and $\omega_{0 e}$ are two new small quantities which are absence in Herzberg's level formula [9]. The Algebraic method (AM) determined diatomic molecular vibrational energy level was put forwarded by Sun Weiguo [14]. Using method, Equation (1) can be rewritten as an algebraic equation

$$
A X=E
$$

where the spectrum data solution matrix $X$ and the level matrix $E$ are

$$
X_{e}=\left(\begin{array}{c}
\omega_{0} \\
\omega_{e}^{\prime} \\
\vdots \\
\omega_{e} r_{e}
\end{array}\right) \quad E=\left(\begin{array}{c}
E_{1} \\
E_{2} \\
\vdots \\
E_{8}
\end{array}\right)
$$

The matrix A is an $8 \times 8$ coefficient square, its matrix element is $A_{v k}=(v+0.5)^{k}$. A group of vibrational energy level, whose number is $m \geq 8$, can be obtained with modern experimental technologies, chosen eight vibrational levels from $m$ levels are instituted into Equation (2), a set of vibrational spectrum constants $X$ can be solved using the standard algebraic method. There are $N=C_{m}^{8}$ different selection schemes, which give $N$ matrixes of vibration spectrum constant $X_{e}$. In the resolved matrixes, there is always a group of vibration spectrum data can best meet with the requirements of the following physical conditions [14].

$$
\Delta E_{v_{\max }, v_{\max -1}}=E_{v_{m}}-E_{v_{m}-1} \rightarrow \text { as small as possible }
$$




$$
\begin{aligned}
& \overline{\Delta E(e, c)}=\sqrt{\frac{1}{m} \sum_{v=0}^{m-1}\left|E_{v, \text { exp }}-E_{v, c a l}\right|^{2}} \rightarrow 0 \\
& E_{v_{\max }} \leq D_{e} \\
& D_{e}^{c a l} \cong E_{v_{\max }}+\Delta E_{v_{\max }, \nu_{\max }-1}^{2} /\left(\Delta E_{v_{\max }, v_{\max 2}}-\Delta E_{v_{\max }, v_{\max }-1}\right) \\
& 0<E_{\text {rror }}=\left(D_{e}^{\exp }-D_{e}^{c a l}\right) / \Delta E_{v_{\max }, v_{\max }-1} \leq 1
\end{aligned}
$$

In 2008, a full set $\left\{E_{v}^{A M}\right\}$ of vibrational levels, the highest vibrational quantum number $v_{\max }$ and dissociation energy $D_{e}$ of HF in $\mathrm{X}^{1} \Sigma^{+}$state have been obtained by Liu Yi-Ding, Sun Weiguo et al. with AM method [17]. The AM data and experiment data [15] are all listed in Table 1.

The full set $\left\{E_{v}^{A M}\right\}$ of vibrational levels and maximum vibration quantum number $v_{\max }$ determined with AM method from the finite and precision experimental vibrational levels are the important basis on the study of macroscopic thermodynamic properties of real gas HF.

\section{Statistical Methods and Results}

The core and important basis of quantum statistical theory are distribution function of statistical ensemble. In this study, the set energy level $\left\{E_{v}^{A M}\right\}$ of HF is not analytical but numerical, the number of real vibration levels is limited but unlimited. When vibration quantum number $v$ is greater than the $v_{\max }$, the corresponding vibrational levels does not actually exist. In calculating the partition function, these vibrational levels should be not included in the sum of vibrational levels. Using the canonical quantum ensemble, the vibrational partition function

Table 1. Comparisons of different vibrational energy spectra of $\mathrm{HF}$ in $\mathrm{X}^{1} \Sigma^{+}$(in $\mathrm{cm}^{-1}$ ).

\begin{tabular}{ccc}
\hline$v$ & exp $[15]$ & $A M$ \\
\hline 0 & 2050.771 & 2050.771 \\
1 & 6012.194 & 6012.194 \\
2 & 9801.56 & 9801.895 \\
3 & 13423.603 & 13424.012 \\
4 & 16882.448 & 16882.721 \\
5 & 20181.824 & 20181.824 \\
6 & 23324.620 & 23324.482 \\
7 & 26313.146 & 23613.042 \\
8 & 29148.927 & 29148.927 \\
9 & 31832.367 & 31832.531 \\
10 & 34362.909 & 34363.093 \\
11 & 36738.405 & 36738.494 \\
12 & 38954.943 & 38954.943 \\
13 & 41006.593 & 41006.508 \\
14 & 42884.443 & 42884.443 \\
15 & 44576.055 & 44576.279 \\
16 & 46064.207 & 46064.629 \\
17 & 47325.663 & 47325.663 \\
18 & 48328.541 & 48327.221 \\
19 & 49026.508 & 49026.508 \\
20 & & 49367.340 \\
$D_{e}^{\text {exp }}$ & & 49380.000 \\
\hline & &
\end{tabular}


is [3]

$$
Q(T)=\sum_{v=0}^{\nu_{\max }} g_{v} e^{-\beta E_{v}}
$$

where $g_{v}(=1)$ is the degeneracy of vibrational level of HF in ground state, $k$ is Boltzmann constant and $\beta=1 / k T$. Molar heat capacity is [3]

$$
C_{m}^{v}=N_{A} k \frac{\partial}{\partial T}\left[T^{2} \frac{\partial}{\partial T} \ln Q(T)\right]
$$

In statistical physics, molar heat capacity is an important observable quantity. For HF macro system, as temperature is not very high, the thinking may be reasonable that the vibration of HF in electronic ground state makes only real contribution for vibrational heat capacity. A kind of common practice was that simple harmonic oscillator (SHO) model was used, and sum was over infinite vibrational levels, the operation is equivalent with $v_{\max } \rightarrow \infty$, and the sum is deservedly changed into integral. So thus obtained partition function is analytical form [3]

$$
Q_{S H O}^{c a l}(T)=\frac{e^{-\beta \hbar \omega_{e} / 2}}{1-e^{-\beta \hbar \omega_{e}}}
$$

The result leads to analytical vibrational heat capacity.

$$
C_{v, S H O}^{c a l}=N_{A} k\left(\beta \hbar \omega_{e}\right)^{2} \frac{e^{\beta \hbar \omega_{e}}}{\left(e^{\beta \hbar \omega_{v}}-1\right)^{2}}
$$

where $N_{A}$ is Avogadro constant. $R\left(=k N_{A}\right)$ is universal gas constant.

In fact, it is not reasonable for simple harmonic oscillator (SHO) model to be used to discuss thermodynamic properties of real macro system, $v_{\max }$ is not infinite. As real number of diatomic molecular vibration levels is generally dozens or even hundreds, so that it hard to obtain analytical solution of thermodynamic properties, and the calculations are usually complex. For example, AM method have been used to calculate full vibrational energy spectrum $\left\{E_{v}\right\}$ and maximum vibrational quantum number $v_{\max }$ of HF in ground state, obtained $v_{\max }=20$. Molar internal energy, entropy, heat capacity and other macroscopic thermodynamic properties of statistical system made of HF in ground state can be obtained. But the results are not equal to one's of summation over infinite items. For example, molar vibrational heat capacity should be expressed as [3]

$$
C_{v, A M}^{c a l}=\frac{N_{A}}{k T^{2}}\left(<E_{v}^{2}>-<E_{v}>^{2}\right)
$$

where $\left\langle E_{v}>\right.$ is statistical average of finite vibrational levels and $\left\langle E_{v}^{2}\right\rangle$ is statistical average of square of finite vibrational levels, they are respectively decided by the following formulas [3]

$$
\begin{aligned}
& <E_{v}>=\frac{\sum_{v=0}^{\max } g_{v} E_{v} e^{-\beta E_{v}}}{\sum_{v=0}^{\max } g_{v} e^{-\beta E_{v}}} \\
& <E_{v}^{2}>=\frac{\sum_{v=0}^{\max } g_{v} E_{v}^{2} e^{-\beta E_{v}}}{\sum_{v=0}^{\max } g_{v} e^{-\beta E_{v}}}
\end{aligned}
$$

Based on the theorv of auantum statistical ensemble, using above the accurate full set of $\left\{E_{v}^{A M}\right\}$ vibrational energy spectrum and $v_{\max }$ of HF in ground state, its vibrational heat capacity $C_{v, A M}^{c a l}$ can be obtained.

\section{Theoretical Results and Comparison}

Hydrogen fluoride is colorless gas. It is the principal industrial source of fluorine, component of many superacids and precursor to many important compounds including pharmaceuticals and polymers. Study on the heat 
capacity of hydrogen fluoride gas is important.

To test the correctness of theoretical results, these results will be compared with reliable experimental data. In this work, reference [18] gives experimental heat capacity (in constant pressure) data of HF gas. To convert these data to vibrational molar heat capacity $C_{v}^{\exp }$ and to reduce complicacies from intermolecular interactions in actual gas, the chosen temperature range $(1000 \mathrm{~K} \sim 3000 \mathrm{~K})$ should be suitable to ideal gas that there are not obvious errors in relevant data, the translational and rotational heat capacities have been deducted.

The three kinds of vibrational heat capacities $C_{v, A M}^{c a l}, C_{v, S H O}^{c a l}$ and $C_{v}^{\exp }$ (their unit are $\mathrm{J} / \mathrm{mol} \cdot \mathrm{K}$ ) and calculated relative percentage error $\delta_{\mathrm{AM}}$ and $\delta_{\mathrm{SHO}}$ are all listed in Table 2. In given temperature range, the SHO heat capacity $C_{v, S H O}^{c a l}$ goes prematurely into the expected scope of the classical energy equipartition theorem, AM vibrational heat capacities $C_{v, A M}^{c a l}$ and experimental heat capacities $C_{v}^{\text {exp }}$ are much smaller than SHO vibrational heat capacities $C_{v, \mathrm{SHO}}^{\mathrm{cal}}$. At $1000 \mathrm{~K}$, the relative error $\delta_{\mathrm{SHO}}$ is as high as $659.12 \%$, heat capacity $C_{v, \mathrm{SHO}}^{\mathrm{cal}}$ is too much larger than theoretical $C_{v, A M}^{c a l}$ and experimental $C_{v}^{\exp }$, but the relative error $\delta_{A M}$ is only $-14.59 \%$. At $2000 \mathrm{~K}, \delta_{S H O}$ is still as high as $68.38 \%$, the relative error $\delta_{A M}$ has been down to $-7.56 \%$. The relative errors $\delta_{S H O}$ are so large that the $C_{v, S H O}^{c a l}$ can not be used in all cases. On the whole, AM $C_{v, A M}^{c a l}$ data are qualitative agreement with experimental $C_{v}^{\text {exp }}$ data. Relative errors $\delta_{A M}$ may be acceptable for actual works. Figure 1 obviously and visually represents the differences and change trends bring three kinds of vibration heat capacity, "*”, “ $\mathbf{\Lambda}$ ” and " $O$ " are $C_{v, A M}^{c a l}, C_{v, S H O}^{c a l}$ and $C_{v}^{\exp }$, respectively. As shown in Figure 1, the vibrational heat capacities from AM full vibrational levels set $\left\{E_{v}\right\}$ are obviously superior to one's of SHO's.

It is easy to see that the rough potential energy function and the summation over infinite levels led to deviation from real situation. The differences from heat capacities $C_{v, A M}^{c a l}$ determined by limited AM vibrational levels with experimental data are obviously superior to one's of SHO. But relative errors are still bigger. There can be the causes, molecular rotation may be non-rigid, the coupling of molecular vibration—rotation are not considered, some molecules are excited. The problems will be discussed in other papers.

Table 2. Comparisons of different vibrational heat capacities.

\begin{tabular}{|c|c|c|c|c|c|}
\hline $\mathrm{T} 10^{3} \mathrm{~K}$ & $C_{v, A M}^{c a l}$ & $C_{\nu, \mathrm{SHO}}^{\mathrm{cal}}$ & $C_{v}^{\exp }$ & $\delta_{A M} \%$ & $\delta_{\text {SHO }} \%$ \\
\hline 1.0 & 1.103 & 8.115 & 1.069 & -14.59 & 659.12 \\
\hline 1.1 & 1.275 & 8.149 & 1.459 & -12.61 & 458.53 \\
\hline 1.2 & 1.672 & 8.176 & 1.929 & -13.84 & 323.85 \\
\hline 1.3 & 2.061 & 8.196 & 2.369 & -13.00 & 245.97 \\
\hline 1.4 & 2.458 & 8.212 & 2.789 & -11.87 & 199.44 \\
\hline 1.5 & 2.846 & 8.225 & 3.189 & -10.76 & 157.92 \\
\hline 1.6 & 3.219 & 8.236 & 3.559 & -9.55 & 131.41 \\
\hline 1.7 & 3.573 & 8.245 & 3.919 & -8.83 & 110.39 \\
\hline 1.8 & 3.906 & 8.252 & 4.259 & -8.29 & 93.75 \\
\hline 1.9 & 4.218 & 8.259 & 4.579 & -7.88 & 80.37 \\
\hline 2.0 & 4.510 & 8.264 & 4.879 & -7.56 & 68.38 \\
\hline 2.1 & 4.781 & 8.269 & 5.169 & -7.51 & 59.97 \\
\hline 2.2 & 5.033 & 8.273 & 5.449 & -7.63 & 51.83 \\
\hline 2.3 & 5.267 & 8.276 & 5.709 & -7.74 & 44.96 \\
\hline 2.4 & 5.485 & 8.279 & 5.949 & -7.80 & 39.17 \\
\hline 2.5 & 5.687 & 8.282 & 6.179 & -7.96 & 34.03 \\
\hline 2.6 & 5.867 & 8.285 & 6.399 & -8.31 & 29.47 \\
\hline 2.7 & 6.050 & 8.287 & 6.609 & -8.46 & 25.39 \\
\hline 2.8 & 6.213 & 8.289 & 6.799 & -8.62 & 21.92 \\
\hline 2.9 & 6.366 & 8.290 & 6.989 & -8.91 & 18.61 \\
\hline 3.0 & 6.508 & 8.292 & 7.159 & -9.09 & 15.83 \\
\hline
\end{tabular}




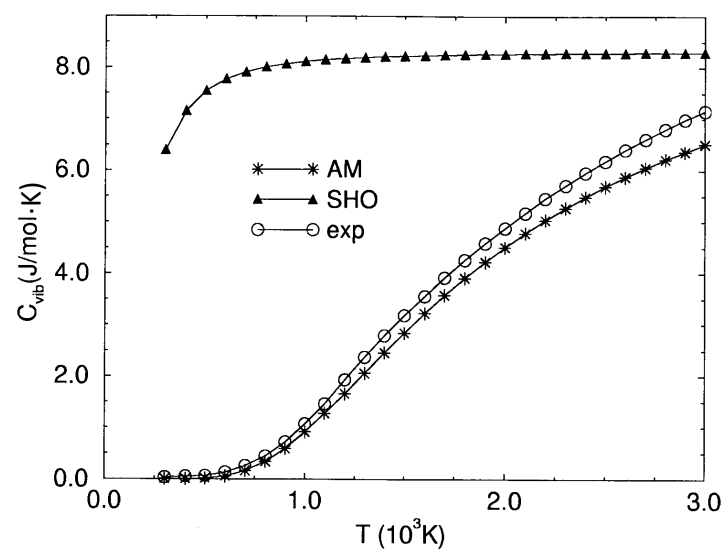

Figure 1. Comparison of heat capacities $C_{v i}$.

\section{Acknowledgements}

This work is supported by the science Foundation of Department of Science and Technology of Sichuan Province (2013JY0077).

\section{References}

[1] Ambaye, H. and Manson, J.R. (2006) Calaulations of Accommodation Coefficients for Diatomic Molecular Gases. Physical Review E, 73, 031202.

[2] Tandon, P. and Uttam, K.N. (2009) Thermodyamic Properties of Platinum Diatomics. Platinum Metals Review, 53, 123-134. http://dx.doi.org/10.1595/147106709X463688

[3] Pathria, R.K. (1977) Statistical Mechanics. 2nd Edition, Pergamon Press, London, 100-107.

[4] Morse, P.M. (1929) Diatomic Molecules According to Wave Mechanics. II . Vibrational Levels. Physical Review, 34, 57-60.

[5] Murrell, J.N. and Sorbie, K.S. (1974) New Analytic Form for the Potential Energy Curves of Stable Diatomic States. Journal of the Chemical Society, Faraday Transactions 2, 70, 1552-1557.

[6] Sun, W.G. (1997) The Energy-Consistent Method for the Potential Energy Curves and the Vibrational Eigenfunction of Stable Diatomic States. Molecular Physics, 92, 105-109. http://dx.doi.org/10.1080/00268979709482078

[7] Sun, W.G. and Feng, H. (1999) An Energy Consistent Method for Potential Energy Curves of Diatomic Molecules. Journal of Physics B, 32, 109-5113.

[8] Dunham, J.L. (1932) The Energy Levels of a Rotating Vibrator. Physical Review, 41, 721-731. http://dx.doi.org/10.1103/PhysRev.41.721

[9] Herzberg, G. (1953) Molecular Spectra and Molecular Structure (I), Spectra of Diatomic Molecules. Van Nostrand, New York, 92.

[10] Bytautas, L., Matsunaga, N., Scuseria, G.E. and Ruedenberg, K. (2012) Accurate Potential Energy Curve for $B_{2} A b$ Initio Elucidation of Experimentally Elusive Ground State Rotation-Vibration Spectrum. The Journal of Physical Chemistry A, 116, 1717-1729. http://dx.doi.org/10.1021/jp210473e

[11] Redberg, R. (1931) The Ro-Vibrational Energy Levels of Diatomic Olecules. Zeitschrift für Physik, 73, 376-385.

[12] Klein, O. (1932) Zur berechnung von Potential kurven für zweiatominge molecule. Zeitschrift für Physik, 76, $226-334$. http://dx.doi.org/10.1007/BF01341814

[13] Rees, A.L.G. (1947) The Calculation of Potential-Energy Curves from Band-Spectroscopoc Data. Proceedings of the Physical Society, 59, 998-1010. http://dx.doi.org/10.1088/0959-5309/59/6/310

[14] Sun, W.G., Hou, S., Feng, H. and Ren, W.Y. (2002) Studies on the Vibrotional and Ro-Vibratioal Energies and Vibrational Force Constants of Diatomic Molecular States Using Algebraic and Variational Methods. Journal of Molecular Spectroscopy, 215, 93-105. http://dx.doi.org/10.1006/jmsp.2002.8619

[15] Coxon, J.A. and Hajigeorgiou, P.G. (1990) Isotopic Dpendence of Born Oppenheimer Breakdown Effects in Diatomic Hydrides: $B^{1} \Sigma^{+}$and $\mathrm{X}^{1} \Sigma^{+}$States of HF and DF. Journal of Molecular Spectroscopy, 142, 254-278. http://dx.doi.org/10.1016/0022-2852(90)90182-P 
[16] Meyer, W. and Rosmus, P. (1975) PNO-CI and CEPA Studies of Electron Correlation Effect.III. Spectroscopic Constants and Dipole Moment Functions for the Ground States of the First-Row and Second-Row Diatomic Hydrides. The Journal of Chemical Physics, 63, 2356-2375.

[17] Liu, Y.D., Sun, W.G. and Zhang, J.P. (2008) Studies on the Full Vibrational Energy Spectra and Potential Energy Curves for Ground State of Fluorine Hydrogen $\mathrm{HF}$ and Its Cation $\mathrm{HF}^{+}$. Journal of Sichuan University (Natural science Edition), 45, 864-868 (Chinese)

[18] Chase Jr., M.W. (1998) NIST-JANAF Themochemical Tables. 4th Edition, J. Phys. Chem. Ref. Data, Monograph 9, $1-1951$. 